\title{
Qualitative assessment of the role of public health education program on HIV transmission dynamics
}

\author{
N. HUSSAINI AND M. WINTER †* \\ Department of Mathematical Sciences, Brunel University, \\ West London, UB8 3PH, UK
}

AND

\author{
A. B. GUMEL \\ Department of Mathematics, University of Manitoba, Winnipeg, \\ Manitoba, R3T 2N2, Canada
}

\begin{abstract}
This paper presents a nonlinear deterministic model for assessing the impact of public health education campaign on curtailing the spread of the HIV pandemic in a population. Rigorous qualitative analysis of the model reveals that it exhibits the phenomenon of backward bifurcation (BB), where a stable disease-free equilibrium coexists with a stable endemic equilibrium when a certain threshold quantity, known as the effective reproduction number $\left(\mathcal{R}_{\text {eff }}\right)$, is less than unity. The epidemiological implication of BB is that a public health education campaign could fail to effectively control HIV, even when the classical requirement of having the associated reproduction number less than unity is satisfied. Furthermore, an explicit threshold value is derived above which such an education campaign could lead to detrimental outcome (increase disease burden),

*Corresponding author Email: matthias.winter@brunel.ac.uk
\end{abstract}


and below which it would have positive population-level impact (reduce disease burden in the community). It is shown that the BB phenomenon is caused by imperfect efficacy of the public health education program. The model is used to assess the potential impact of some targeted public health education campaigns using data from numerous countries.

Keywords: HIV/AIDS; Reproduction number; Stability; Equilibria; Backward bifurcation.

\section{Introduction}

Since its emergence in the 1980s, the human immunodeficiency virus (HIV), and the associated syndrome of opportunistic infections which lead to the late stage HIV disease, known as the acquired immunodeficiency syndrome (AIDS), continues to be one of the most serious global public health menace. Over 33 million people are currently living with HIV (UNAIDS, 2007). Based on the current trends, over 6800 persons become infected with HIV, and 5700 die from AIDS-related causes, every day (UNAIDS, 2007). AIDS is the leading cause of death in sub-saharan Africa, especially in the southern part of the continent. Moreover, $68 \%$ of HIV-related deaths and $76 \%$ of the total new infections occurred in sub-saharan Africa (UNAIDS, 2007). There is still no cure or vaccine for HIV, and anti-retroviral drugs (ARVs) are still not widely accessible, particularly in the resource-poor nations (which suffer the vast majority of the HIV burden globally). Yet, HIV remains preventable through the avoidance of high-risk behaviour, such as unprotected sexual intercourse and sharing of drug injection needles. Thus, in the absence of pharmaceutical interventions (such as a vaccine or ARVs) in areas where the HIV pandemic is more rampant (notably developing nations), the effective control of HIV would depend, primarily, on reducing behavioural risks. This could be 
achieved through effective public heath education campaign.

Unfortunately, however, surveys around the world show alarming low level of awareness and understanding about HIV and its preventive measures (Keitshokil et al., 2007; Pérez et al., 2008). Recent studies indicate that the most effective available means to control the prevalence of HIV is to provide HIV-related education, which will lead to safe lifestyles among sexually-active members of the public (Bortolotti et al., 1992; Morton et al., 1996). Moreover, education, as a sole anti-HIV intervention strategy, may not be sufficient to motivate behaviour change (Berker \& Joseph, 1998). Studies show that public health education increases self-efficacy, which is a determinant for controlling risky behaviour (Lindan et al., 1991). Furthermore, as noted by Cassell et al. (2006), the benefits of new methods of HIV prevention could be jeopardised if they are not accompanied by positive efforts to change risky behaviour. This is in line with the well-known fact that sexual education and awareness of the risk and life-threatening consequences of AIDS can lower the incidence rate in HIV infection (Velesco-Hernandez \& Hsieh, 1994).

Public health education campaigns have been successfully implemented in numerous countries and communities, such as: Uganda, Thailand, Zambia and the US gay community (Daniel \& Rand, 2003; de Walque, 2007). Between 1991-1998, HIV prevalence dramatically declined in Uganda from $21 \%$ to $9.8 \%$ (with a corresponding reduction in non-regular sexual partners by $65 \%$ coupled with greater levels of awareness about HIV/AIDS; Daniel \& Rand, 2003). The Ugandan programme fostered community mobilization towards change in risky behaviour, without increasing stigma (Green et al., 2006; Wilson, 2004). In Zambia, the decline in HIV incidence since early 1990s is attributed to behavioural changes (Fylkesnes, 2001).

There are a number of ways (or strategies) public health education campaigns can be implemented (or targeted) effectively to combat the burden of HIV disease (measured 
in terms of new cases, mortality etc) in a community. This study considers the following targeted strategies:

- targeting adult ("established") sexually-active susceptible individuals only;

- targeting newly-recruited sexually-active susceptible individuals only;

- targeting HIV-infected individuals without clinical AIDS symptoms only; or

- targeting HIV-infected individuals with AIDS symptoms only.

The primary goal of this study is to theoretically determine which of the aforementioned targeted strategies (or combination of strategies) is (are) the most effective in curtailing HIV spread in a community.

A number of mathematical models have been designed and used to study the impact of preventive control strategies on the spread of HIV/AIDS in given populations. Some of these studies have shown that a change in risky behaviour is necessary to prevent raging HIV/AIDS prevalence, even in the presence of a vaccine and/or treatment (see, for instance, Anderson, 1988; Blower \& McLean, 1994; Del Valle et al., 2004; KribsZaleta \& Valesco-Hernandez, 2000). Anderson (1988) predicts rapid transmission of HIV when the infected individuals engage in risky behaviours. Smith \& Blower (2004) reported that disease-modifying vaccines will reduce HIV transmission if they cause a reduction of $1.5 \log _{10}$ copies/mL or more in viral load and if risky behaviours do not increase. The studies mentioned above tend to emphasize the use of pharmaceutical interventions (such as vaccine and ARVs), which are not readily and widely available (especially in resource-poor nations, which constitute the vast majority of the global HIV prevalence). Thus, it is instructive to study models that focus on nonpharmaceutical interventions, such as the use of public health education campaign. A few modelling studies, such as those by Mukandavire et al. (2009), Mukandavire 
and Garira (2007) and Del Valle et al. (2004), have investigated the impact of public health educational campaigns on the transmission dynamics of HIV/AIDS in some populations. The purpose of the current study is to extend some of the aforementioned studies, by designing and analyzing a new comprehensive model, for HIV transmission in a population, that incorporates the role of public health education campaign (and using the model to evaluate the impact of some targeted public health education strategies).

The paper is structured as follows. The model is formulated and fitted with real data in Section 2. Public health education campaign strategies are assessed, both theoretically and numerically, in Section 3. The existence of backward bifurcation is established in Section 4.

\section{Model Formulation}

The total population at time $t$, denoted by $N(t)$, is sub-divided into the following mutually exclusive sub-populations: uneducated susceptible individuals $\left(S_{u}(t)\right)$, educated susceptible individuals $\left(S_{e}(t)\right)$, uneducated infected individuals with no AIDS symptoms $\left(I_{u}(t)\right)$, educated infected individuals with no AIDS symptoms $\left(I_{e}(t)\right)$, uneducated infected individuals with AIDS symptoms $\left(A_{u}(t)\right)$ and educated infected individuals with AIDS $\left(A_{e}(t)\right)$. Here, (un)educated means individuals who (do not) receive proper public health education or counseling against risky practices that may result in HIV infection. The model takes the form of the following deterministic system of nonlinear differential equations: 


$$
\begin{aligned}
\frac{d S_{u}}{d t} & =\Pi(1-p)-\xi S_{u}-\left[\lambda_{u}+(1-\kappa) \lambda_{e}\right] S_{u}-\mu S_{u}, \\
\frac{d S_{e}}{d t} & =\Pi p+\xi S_{u}-(1-\epsilon)\left[\lambda_{u}+(1-\kappa) \lambda_{e}\right] S_{e}-\mu S_{e}, \\
\frac{d I_{u}}{d t} & =\left[\lambda_{u}+(1-\kappa) \lambda_{e}\right] S_{u}-\sigma_{u} I_{u}-\mu I_{u}-\psi_{1} I_{u}, \\
\frac{d A_{u}}{d t} & =\sigma_{u} I_{u}-\psi_{2} A_{u}-\mu A_{u}-\delta_{u} A_{u}, \\
\frac{d I_{e}}{d t} & =(1-\epsilon)\left[\lambda_{u}+(1-\kappa) \lambda_{e}\right] S_{e}+\psi_{1} I_{u}-\sigma_{e} I_{e}-\mu I_{e} \\
\frac{d A_{e}}{d t} & =\sigma_{e} I_{e}+\psi_{2} A_{u}-\mu A_{e}-\delta_{e} A_{e},
\end{aligned}
$$

where,

$$
\lambda_{u}=\frac{\beta\left(I_{u}+\eta_{u} A_{u}\right)}{N} \text { and } \quad \lambda_{e}=\frac{\beta\left(I_{e}+\eta_{e} A_{e}\right)}{N} .
$$

The rates $\lambda_{u}$ and $\lambda_{e}$ above are the forces of infection associated with HIV transmission by uneducated (at the rate $\lambda_{u}$ ) and educated (at the rate $\lambda_{e}$ ) infected individuals, respectively. The parameter $\beta$ is the effective contact rate (that is, contact that may result in HIV infection), while the parameters $\eta_{u}>\eta_{e}>1$ account for the relative infectiousness of individuals with AIDS symptoms in comparison to the corresponding infected individuals with no AIDS symptoms. Unlike in the other related modelling studies, such as those by Mukandavire et al. (2009), Mukandavire \& Garira (2007) and Del Valle et al. (2004), this study allows for the transmission of HIV by individuals with AIDS symptoms (in line with Elbasha \& Gumel, 2006 and also Garba \& Gumel, 2010).

Recruitment into the sexually-active population occurs at a rate $\Pi$ (all newlyrecruited individuals are assumed to be susceptible to HIV infection), and a fraction, $p$, of these newly-recruited sexually-active individuals are assumed to be educated about 
the risks and consequences of the HIV disease. Uneducated susceptible individuals (excluding the newly-recruited individuals) receive education about safer sex practices at a rate $\xi$. Susceptible people acquire infection following effective contact with infected individuals (at the rates $\lambda_{u}$ and $\lambda_{e}$ ). It is assumed that educated infected individuals (in $I_{u}$ or $A_{u}$ class) modify their behaviour positively, thereby reducing their risk of HIV transmission by a factor $\kappa$, with $0<\kappa<1$. In other words, it is assumed that HIV-infected individuals that received public health education transmit the disease at a lower rate in comparison to uneducated HIV infected individuals. Educated susceptible individuals acquire infection at a reduced rate $(1-\epsilon)\left[\lambda_{u}+(1-\kappa) \lambda_{e}\right]$, where $0<\epsilon<1$ is the efficacy of public health education in preventing new infection of educated susceptible individuals.

Uneducated infected individuals progress to AIDS at a rate $\sigma_{u}$, while educated infected individuals progress at a reduced rate $\sigma_{e}<\sigma_{u}$ (in other words, infected individuals who received public health education progress to AIDS at a slower rate in comparison to those who do not). Uneducated infected individuals without AIDS symptoms $\left(I_{u}\right)$ are educated at a rate $\psi_{1}$, and move to the corresponding educated infected class $\left(I_{u}\right)$. Individuals in all classes suffer natural death at a rate $\mu$. Additionally, individuals with AIDS die at a rate $\delta_{u}$ (for the uneducated class) or $\delta_{e}$ (for the educated class) such that $\delta_{e}<\delta_{u}$. Thus, it is assumed that AIDS patients who received public health education die due to AIDS at a slower rate than the AIDS patients who do not. Uneducated individuals with symptoms of AIDS $\left(A_{u}\right)$ are educated at a rate $\psi_{2}$, and move to the corresponding educated class $\left(A_{e}\right)$. A schematic diagram of the model is depicted in Figure 1, and the associated variables and parameters are described in Table 1.

The model (1) is an extension of the models by Mukandavire et al. (2009), Mukandavire \& Garira (2007) and Del Valle et al. (2004), by 
(i) allowing for HIV transmission by the individuals with AIDS symptoms;

(ii) offering public health education to all infected individuals (except for the education of high-risk people with AIDS in Mukandavire and Garira, 2007; public health education is only restricted to susceptible individuals in Mukandavire et al., 2009; and Del Valle et al., 2004);

(iii) stratifying the infected population in terms of whether or not they received public health education (and those who received public health education are assumed to transmit HIV at a lower rate, as well as progress to AIDS and die at a slower rate, in comparison to those who do not receive public health education).

(iv) The model extends the model by Garba \& Gumel, 2010 by including a class of susceptible individuals who receive public health education, educating a fraction of newly-recruited sexually-active individuals and allowing infection of educated susceptible individuals. Furthermore, in this study, the infected individuals who received public health education progress to AIDS at a slower rate in comparison to those who do not.

In addition to the aforemention extensions, this study will contribute to the literature by giving detailed qualitative analysis of the model (1).

\subsection{Model Fitting}

To test the suitability of the model (1) to effectively enable the assessment of targeted public health education strategies against HIV spread in a population, the model is fitted using data from Uganda as follows. The average lifespan of a Ugandan $(1 / \mu)$ is assumed to be 50 years (UBSC, 1991) and the recruitment rate (П) is estimated at $3.2 \%$ of the total population (UBSC, 1991). The total population of Uganda, as of 1990, given by $N=16.7$ millions (UBSC, 1991) is used. The initial conditions used are 
as follows: $S_{u}(0)=14$ million, $S_{e}(0)=0.4121$ million, $I_{u}(0)=2$ million, $A_{u}(0)=0.2$ million, $I_{e}(0)=0.087$ million, and $A_{e}(0)=0.0009$ million. Thus, the total initial HIVinfected population (i.e., $\left.I_{u}(0)+A_{u}(0)+I_{e}(0)+A_{e}(0)\right)$ is 2.2879 million (UNAIDS, 2008), corresponding to $13.7 \%$ of the total population. The associated epidemiological data is presented in Table 2 .

Using the aforementioned data, the model (1) gives a very good fit of the Ugandan HIV/AIDS data for the period 1990-2007 (UNAIDS, 2008; UNAIDS/WHO/Unicef, 2008), as depicted in Figure 2. Furthermore, to qualitatively assess the closeness of the model against the real data, Ordinary Least Squares (OLS) approach is employed (Kendall \& Stuart, 1979). This entails regressing the actual observed data on predicted cases from the model as follows.

Let $y_{\text {obs }}$ denotes the observed data. Then, the model prediction $\left(\hat{y}_{\text {pred }}\right)$ is evaluated using the OLS regression equation:

$$
y_{\text {obs }}=\alpha_{0}+\alpha_{1} \hat{y}_{\text {pred }}+\varepsilon,
$$

where $\alpha_{0}$ and $\alpha_{1}$ represent the intercept and slope of the regression line, respectively; and $\varepsilon$ account for the random error. The model is said to be "perfect" if the coefficients $\alpha_{0}=0$ and $\alpha_{1}=1$ and the coefficient of determination $R^{2}=1$ (which measures the proportion of variation in the $\left.y_{o b s}\right)$. Using MATLAB's Statistical Toolbox, we obtained $\alpha_{0}=0.0636$ and $\alpha_{1}=0.9603$ (with their corresponding

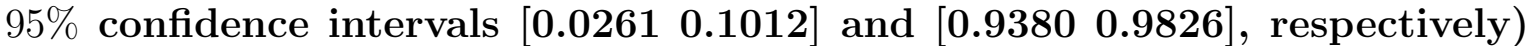
and $R^{2}=0.9981$ for the above initial data and parameter values in Table 2 and 3. Thus, the OLS regression analysis confirms the closeness of the fit. Hence, the model (1) can be used to gain realistic insight into HIV transmission dynamics in the presence of public health education campaign. 


\section{Model Analysis}

Since the model (1) monitors human population, all its associated parameters and state variables are assumed to be non-negative for all $t \geq 0$. Before analysing the model, it is instructive to show that the state variables of the model remain non-negative for all non-negative initial conditions. Thus, we claim the following result.

Lemma 1. The closed set

$$
\mathcal{D}=\left\{\left(S_{u}, S_{e}, I_{u}, A_{u}, I_{e}, A_{e}\right) \in \mathbb{R}_{+}^{6}: N \leq \frac{\Pi}{\mu}\right\}
$$

is positively-invariant and attracting with respect to the model (1).

Proof. Adding all the equations in the model (1) gives:

$$
\frac{d N}{d t}=\Pi-\mu N-\delta_{e} A_{e}-\delta_{u} A_{u}, \quad \text { where } \quad N=S_{u}+I_{u}+A_{u}+S_{e}+I_{e}+A_{e} .
$$

Since $\frac{d N(t)}{d t} \leq \Pi-\mu N$, it follows that $\frac{d N(t)}{d t}<0$ if $N(t)>\frac{\Pi}{\mu}$. Thus, a standard comparison theorem (see Lakshmikantham et al., 1989) can be used to show that $N(t) \leq N(0) e^{-\mu t}+\Pi / \mu\left(1-e^{-\mu t}\right)$. In particular, $N(t) \leq \Pi / \mu$ if $N(0) \leq \Pi / \mu$. Thus, $\mathcal{D}$ is positively-invariant. Further, if $N(t)>\frac{\Pi}{\mu}$, then either the solution enters $\mathcal{D}$ in finite time, or $N(t)$ approaches $\Pi / \mu$. Hence, $\mathcal{D}$ is attracting (i.e., all solutions in $\mathbb{R}_{+}^{6}$ eventually approach, enter or stay in $\mathcal{D}$ ).

Therefore, the model is mathematically well-posed and epidemiologically reasonable, since all the variables remain nonnegative for all $t \geq 0$. Hence, it is sufficient to consider the dynamics of the model (1) in $\mathcal{D}$ (Hethcote, 2000). 


\subsection{Local stability of Disease-free equilibrium (DFE)}

The model (1) has a unique disease-free equilibrium, obtained by setting the right-hand sides of the equations in the model (1) to zero, given by

$$
\mathcal{X}=\left(S_{u}^{*}, S_{e}^{*}, I_{u}^{*}, A_{u}^{*}, I_{e}^{*}, A_{e}^{*}\right)=\left[\frac{\Pi(1-p)}{\xi+\mu}, \frac{\Pi(p \mu+\xi)}{\mu(\xi+\mu)}, 0,0,0,0\right]
$$

It can be shown that $\mathcal{X}$ attracts the region (the stable manifold of $\mathcal{X}$ )

$$
\mathcal{D}_{\mathcal{X}}=\left\{\left(S_{u}, S_{e}, I_{u}, A_{u}, I_{e}, A_{e}\right) \in \mathcal{D}: I_{u}=A_{u}=I_{e}=A_{e}=0\right\}
$$

Using the next generation operator method (van den Driessche \& Watmough, 2002), the associated matrices $F_{e}$, for the new infection terms, and $V_{e}$, for the remaining transition terms, are, respectively, given by (noting that $N^{*}=\frac{\Pi}{\mu}$ at $\mathcal{X}$ )

$$
F_{e}=\left(\begin{array}{cccc}
\beta \frac{S_{u}^{*}}{N^{*}} & \eta_{u} \beta \frac{S_{u}^{*}}{N^{*}} & \beta(1-\kappa) \frac{S_{u}^{*}}{N^{*}} & \beta(1-\kappa) \eta_{e} \frac{S_{u}^{*}}{N^{*}} \\
0 & 0 & 0 & 0 \\
\beta(1-\epsilon) \frac{S_{e}^{*}}{N^{*}} & \beta(1-\epsilon) \frac{S_{e}^{*}}{N^{*}} \eta_{u} & \beta(1-\kappa)(1-\epsilon) \frac{S_{e}^{*}}{N^{*}} & \beta(1-\kappa)(1-\epsilon) \eta_{e} \frac{S_{e}^{*}}{N^{*}} \\
0 & 0 & 0 & 0
\end{array}\right)
$$

18 and,

$$
V_{e}=\left(\begin{array}{cccc}
K_{1} & 0 & 0 & 0 \\
-\sigma_{u} & K_{2} & 0 & 0 \\
-\psi_{1} & 0 & K_{3} & 0 \\
0 & -\psi_{2} & -\sigma_{e} & K_{4}
\end{array}\right)
$$

where,

$$
K_{1}=\mu+\sigma_{u}+\psi_{1}, \quad K_{2}=\mu+\delta_{u}+\psi_{2}, \quad K_{3}=\mu+\sigma_{e} \quad \text { and } \quad K_{4}=\mu+\delta_{e}
$$

220 It follows that the effective reproductive number, denoted by $R_{\text {eff }}$, is given by 


$$
\mathcal{R}_{e f f}=\rho\left(F_{e} V_{e}^{-1}\right)=\frac{\beta(A+B+C)}{K_{1} K_{2} K_{3} K_{4}(\xi+\mu)},
$$

where $\rho$ is the spectral radius, and

$$
\begin{aligned}
A & =K_{1} K_{2}(1-\epsilon)(1-\kappa)(p \mu+\xi)\left(K_{4}+\eta_{e} \sigma_{e}\right), \\
B & =\mu K_{4} K_{3}(1-p)\left(K_{2}+\sigma_{u} \eta_{u}\right), \\
C & =\mu(1-p)(1-\kappa)\left(\psi_{1} K_{2} K_{4}+\psi_{2} \sigma_{u} K_{3} \eta_{e}+\sigma_{e} \eta_{e} \psi_{1} K_{2}\right) .
\end{aligned}
$$

Biologically-speaking, the effective reproduction number measures the average number of new infections generated by a single HIV infected person in a community where a public health enlightenment campaign is used as a control strategy (Anderson \& May, 1991; Hethcote, 2000; van den Driessche \& Watmough, 2002). Moreover, in the absence of public health education $\left(I_{e}=A_{e}=p=\kappa=\delta_{e}=\xi=\epsilon=\sigma_{e}=\psi_{1}=\psi_{2}=\right.$ $0)$, the quantity $\mathcal{R}_{\text {eff }}=\frac{\beta\left(\mu+\delta_{u}+\eta_{u} \sigma_{u}\right)}{\left(\sigma_{u}+\mu\right)\left(\mu+\delta_{u}\right)}=\mathcal{R}_{0}$, where $\mathcal{R}_{0}$ is the basic reproduction number (i.e., $\mathcal{R}_{0}$ represents the average number of new cases generated by a single infected individual in a completely susceptible population).

Using Theorem 2 of van den Driessche \& Watmough (2002), the following result is established.

Theorem 1. The DFE, $\mathcal{X}$, of the system (1), given by (6), is locally asymptotically stable $(L A S)$ if $\mathcal{R}_{\text {eff }}<1$, and unstable if $\mathcal{R}_{\text {eff }}>1$.

Theorem 1 implies that HIV can be eliminated from the community when $\mathcal{R}_{\text {eff }}<1$, provided the initial sizes of the sub-populations of the model (1) are within the domain of attraction of $\mathcal{X}$. To ensure that HIV elimination is independent of the initial sizes of the sub-populations, we need to show that the DFE is globally asymptotically stable (GAS). This is established in Section 4, for the special case where the efficacy of public health education is assumed to be $100 \%$ (i.e., $\epsilon=1$ ). 


\subsection{Assessment of Impact of Public Health Education}

Before using the model (1) to assess the impact of public health education in combatting HIV spread in a population, it is instructive to assess the behaviour of the model under the worst case scenario (i.e., the case where no public health education is provided in the community). By setting all education-related parameters to zero (i.e., $p=\kappa=$ $\left.\delta_{e}=\xi=\epsilon=\sigma_{e}=\psi_{1}=\psi_{2}=0\right)$ and using the data in Tables 2 and 3, simulations of the model (1) show that India, Nigeria, China, Ethiopia, and Russia will record around 23.5 million, 12.5 million, 10.1 million, 8.8 million and 6 million total HIV/AIDS cases in eight years, respectively (Figures $3 \mathrm{~A}$ and 3B). These projections of the model (1) are consistent with the estimates given by the US-based National Intelligence Council (2002), which predicts that, by the year 2010, India, Nigeria, China, Ethiopia, and Russia could have about 20 to 25 million, 10 to 15 million, 10 to 15 million, 7 to 10 million, and 5 to 8 million HIV/AIDS cases if the governments of the respective countries do not take serious action against the spread of HIV/AIDS.

\subsubsection{Threshold analysis}

In this section, the impact of public health education campaign will be assessed by carrying out threshold analysis on the effective reproductive number, $\mathcal{R}_{\text {eff }}$, as follows. Let $\omega=\frac{S_{e}^{*}}{N^{*}}$ be the fraction of susceptible individuals educated at the DFE $\mathcal{X}$. Hence, $\mathcal{R}_{\text {eff }}$ can now be rewritten as a function of $\omega$.

$$
\mathcal{R}_{e f f}=\mathcal{R}_{e f f}(\omega)=\frac{\beta\left(Z_{1}+Z_{2}\right)}{K_{1} K_{2} K_{3} K_{4}}
$$


where,

$$
\begin{aligned}
& Z_{1}=\omega K_{1} K_{2}(1-\epsilon)(1-\kappa)\left(K_{4}+\eta_{e} \sigma_{e}\right), \\
& Z_{2}=(1-\omega)\left[(1-\kappa)\left(\psi_{1} K_{2} K_{4}+\psi_{2} K_{3} \sigma_{e} \eta_{e}+\psi_{2} K_{2} \sigma_{u} \eta_{e}\right)+K_{3} K_{4}\left(K_{2}+\eta_{u} \sigma_{u}\right)\right] .
\end{aligned}
$$

Differentiating $\mathcal{R}_{\text {eff }}$, given in (5), partially with respect to $\omega$ gives

$$
\frac{\partial \mathcal{R}_{e f f}(\omega)}{\partial \omega}=-Z_{3}(1-\nabla)
$$

where,

$$
\begin{aligned}
& Z_{3}=\frac{\beta\left[(1-\kappa)\left(\psi_{1} K_{2} K_{4}+\psi_{2} K_{2} \sigma_{e} \eta_{e}+\psi_{2} K_{3} \sigma_{u} \eta_{e}\right)+K_{3} K_{4}\left(K_{2}+\eta_{u} \sigma_{u}\right)\right]}{K_{1} K_{2} K_{3} K_{4}}>0, \\
& \nabla=\frac{K_{1} K_{2}(1-\epsilon)(1-\kappa)\left(K_{4}+\eta_{e} \sigma_{e}\right)}{(1-\kappa)\left(\psi_{1} K_{2} K_{4}+\psi_{2} K_{3} \sigma_{e} \eta_{e}+\psi_{2} K_{2} \sigma_{u} \eta_{e}\right)+K_{3} K_{4}\left(K_{2}+\eta_{u} \sigma_{u}\right)}>0 .
\end{aligned}
$$

Since $Z_{3}$ and $\nabla$ are both non-negative (noting that $0<\kappa<1$ and $0<\epsilon<1$ ), then $\frac{\partial \mathcal{R}_{e f f}(\omega)}{\partial \omega}<0$ whenever $\nabla<1$. Further, $\frac{\partial \mathcal{R}_{e f f}(\omega)}{\partial \omega}>0$ if $\nabla>1$. This result is summarized below.

Lemma 2. The use of public health education campaign would have

(i) a positive population-level impact (reduce disease burden) if $\nabla<1$;

(ii) no population-level impact if $\nabla=1$;

(iii) a detrimental population-level impact (increase disease burden) if $\nabla>1$.

Biologically-speaking, $\nabla$ could be interpreted as the measure of increase or decrease in risky behaviour (or negative attitude) of the individuals in the community who received public health education. That is, $\nabla<1, \nabla=1$ and $\nabla>1$ mean that 
where,

$$
\mathcal{R}_{0}=\frac{\beta\left(\mu+\delta_{u}+\eta_{u} \sigma_{u}\right)}{\left(\sigma_{u}+\mu\right)\left(\mu+\delta_{u}\right)},
$$

and,

$$
\mathcal{R}_{0 e}=\frac{\beta(1-\epsilon)(1-\kappa)\left(K_{4}+\sigma_{e} \eta_{e}\right)}{K_{3} K_{4}} .
$$

$$
\Omega=\frac{\left(\sigma_{u}+\mu\right)\left(\mu+\delta_{u}\right)\left(\gamma_{1}+\gamma_{2}\right)}{\gamma_{3} K_{1} K_{2}(\xi+\mu)\left[K_{1} K_{2} K_{3} K_{4}(\xi+\mu) \mathcal{R}_{0}+\beta(A+B+C)\right]},
$$

where, 


$$
\begin{aligned}
\gamma_{1} & =\mathcal{R}_{0}{ }^{2} K_{1}{ }^{2} K_{2}{ }^{2} K_{3}{ }^{2} K_{4}{ }^{2}(\xi+\mu)^{2}+\beta^{2}(A+B+C)^{2} \\
\gamma_{2} & =\beta K_{3} K_{4}\left(\mu+\delta_{u}+\sigma_{u} \eta_{u}\right)+(1-\epsilon)(1-\kappa)\left(K_{2}+\sigma_{e} \eta_{e}\right)\left(\sigma_{u}+\mu\right)\left(\delta_{u}+\mu\right), \\
\gamma_{3} & =\beta K_{3}^{2} K_{4}^{2}\left(\mu+\delta_{u}+\sigma_{u} \eta_{u}\right)^{2}+(1-\epsilon)^{2}(1-\kappa)^{2}\left(K_{2}+\sigma_{e} \eta_{e}\right)^{2}\left(\sigma_{u}+\mu\right)^{2}\left(\delta_{u}+\mu\right)^{2} .
\end{aligned}
$$

It follows from (7) that the education impact factor (denoted by $\Upsilon$ ) is given by

$$
\Upsilon=\Omega\left(1-\frac{\mathcal{R}_{0 e}}{\mathcal{R}_{0}}\right)
$$

Thus, we have established the following result.

Theorem 2. The use of public health education campaign in the community will have

(i) positive population-level impact if $\Upsilon>0\left(\mathcal{R}_{0 e}<\mathcal{R}_{0}\right)$;

(ii) negative population-level impact in the community if $\Upsilon<0\left(\mathcal{R}_{0 e}>\mathcal{R}_{0}\right)$; and

(iii) no population-level impact in the community if $\Upsilon=0\left(\mathcal{R}_{0 e}=\mathcal{R}_{0}\right)$.

Numerical simulations of the model, using appropriate demographic and epidemiological data for Ethiopia, given in Tables 2 and 3, show the following interesting cases:

$\nabla<1$ : Using the aforementioned realistic set of parameter values (Tables 2 and 3), it follows that $\nabla=0.0517<1, \mathcal{R}_{\text {eff }}=0.6898$ and $\mathcal{R}_{0 e}=0.6619<\mathcal{R}_{0}=1.3712$, so that the use of public health education campaign will have positive populationlevel impact (Figure 4A). In other words, the public health education campaign results in positive behaviour change (in reducing risky practices) in the individuals who received such education (in this case).

$\nabla>1$ : Consider the case with $\xi=0.01, p=\psi_{1}=\psi_{2}=0.001$ and $\epsilon=0.4$ (that is, the coverage rate and efficacy of public health education are low) and all other parameters as above. Here, $\nabla=1.4211>1, \mathcal{R}_{\text {eff }}=1.5866$ and $\mathcal{R}_{0 e}=1.9857>$ $\mathcal{R}_{0}=1.3712$. The simulation results obtained, depicted in Figure $4 \mathrm{~B}$, shows that 
in this setting, the use of public health education increases the number of HIV cases in comparison to the worst-case scenario. This result could be interpreted as follows: the use of "ineffective" public health education campaign (characterize by low coverage and efficacy) induces an increase in risky behaviour amongst people after receiving it.

Contour plots of $\mathcal{R}_{\text {eff }}$ as a function of efficacy of public health education and the fraction of individuals who received public health education (i.e., public health education coverage level) at steady-state are depicted in Figure 5. As expected, an increase in efficacy and coverage level leads to a decrease in $\mathcal{R}_{\text {eff }}$. This is an important result because the main objective of public health education is to reduce $\mathcal{R}_{\text {eff }}$ as much as possible (since reduction in $\mathcal{R}_{\text {eff }}$ is positively correlated with a reduction in disease burden), which could lead to effective disease control or elimination. It is evident from Figure 5 that the prospect of effective control of HIV increases with increasing efficacy and coverage rate of the public health education campaign. For instance, a public health education program with efficacy and coverage level of $60 \%$ (each) will fail to control the disease (since $\mathcal{R}_{\text {eff }}>1$ in this case). On the other hand, the use of public health education campaign with efficacy and coverage level of $90 \%$ (each) could eliminate HIV from the population (see also Figure 7).

\subsection{Evaluation of Targeted Education Strategies}

The model is used to evaluate the impact of the following targeted public health education strategies:

- Strategy I: educating adult ("established") sexually-active susceptible individuals only (at the rate $\xi$ ),

- Strategy II: educating a fraction $p$ newly-recruited sexually-active susceptible 
individuals only,

- Strategy III: educating HIV-infected individuals without clinical AIDS symptoms only (at the rate $\psi_{1}$ ), or

- Strategy IV: educating HIV-infected individuals with clinical AIDS symptoms only (at the rate $\psi_{2}$ ).

Using demographic data from India, Nigeria, China, Ethiopia, and Russia, tabulated in Table 3 ( together with the associated epidemiological data given in Table 2), simulations of model (1) show that Strategy I can prevent more than 0.8642 million, 0.5474 million, 0.3321 million, 0.4064 million, and 0.2116 million new cases in India, Nigeria, China, Ethiopia, and Russia respectively within a year (see Table 4A). Furthermore, Strategy I seems to be the most effective amongst all targeted single group strategies. It is also shown that combining Strategies I and IV gives the most effective strategy for reducing new HIV cases in comparison to all other possible 2-group combined strategies. Moreover, Table 4C shows that the combination of Strategy I, Strategy III and Strategy IV is the best in reducing the total number of new cases than any of the others except the universal strategy (i.e., educating every class of uneducated individuals at a certain rate). The Universal Strategy can prevent more than 1.1590 million, 0.7580 million, 0.3858 million, 0.5731 million, and 0.253 million new cases of HIV in India, Nigeria, China, Ethiopia, and Russia respectively within a year (see Table 4D).

Table 4 further shows that the use of single-group strategy can be more effective than some 3-group or 2-group strategies. For instance, Strategy I is more effective in reducing the number of new infections than the combination of Strategies II, III and IV. Additionally, a 2-group combined strategy can be better in curtailing the number of new cases than a 3-group strategy (this table shows that combining Strategies I and 


$$
G^{* *}=\beta \frac{\left[I_{u}^{* *}+\eta_{u} A_{u}^{* *}+(1-\kappa)\left(I_{e}^{* *}+\eta_{e} A_{e}^{* *}\right)\right]}{N^{* *}}
$$

be the force of infection at an arbitrary equilibrium of (1), denoted by

$$
\mathcal{E}=\left(S_{u}^{* *}, S_{e}^{* *}, I_{u}^{* *}, A_{u}^{* *}, I_{e}^{* *}, A_{e}^{* *}\right)
$$

371 Thus, at steady-state, the equations of the model (1) can be re-written as:

\section{Existence of Backward Bifurcation}

Backward, or subcritical, bifurcation in epidemiological models is typically associated with the co-existence of disease-free equilibrium and endemic equilibria when the $b a$ sic reproduction number $\left(\mathcal{R}_{0}\right)$ is less than unity. This phenomenon has been found in many epidemiological settings (see, for instance, Elbasha \& Gumel, 2006; Hadeler \& van den Driessche, 1997; Kribs-Zaleta \& Valesco-Hernandez, 2000 and the references therein). Furthermore, such phenomenon has been established in a model for public health education campaign by Mukandavire et al., (2009). The epidemiological implication of such a phenomenon is that the classical requirement of having the associated reproduction number less than unity, while necessary is not sufficient condition for disease control. Following the result in Mukandavire et al., (2009), it is instructive to determine whether or not the model (1) also undergoes backward bifurcation. This is explored below.

Let,

IV gives fewer new cases than some 3-group strategies, which include the combination of Strategies I, II and III and also the combination of Strategies II, III and IV). 


$$
\begin{aligned}
& S_{u}^{* *}=\frac{\Pi(1-p)}{\mu+\xi+G^{* *}}, \\
& S_{e}^{* *}=\frac{\Pi\left(p \mu+\xi+p G^{* *}\right)}{\left(\mu+\xi+G^{* *}\right)\left[(1-\epsilon) G^{* *}+\mu\right]}, \\
& I_{u}^{* *}=\frac{\Pi(1-p) G^{* *}}{K_{1}\left(\mu+\xi+G^{* *}\right)}, \\
& A_{u}^{* *}=\frac{\sigma_{u} \Pi(1-p) G^{* *}}{K_{1} K_{2}\left(\mu+\xi+G^{* *}\right)}, \\
& I_{e}^{* *}=\frac{G^{* *} \Pi\left(G^{* *} C^{*}+D^{*}\right)}{K_{1} K_{3}\left(\mu+\xi+G^{* *}\right)\left[(1-\epsilon) G^{* *}+\mu\right]}, \\
& A_{e}^{* *}=\frac{G^{* *} \Pi\left(G^{* *} A^{*}+B^{*}\right)}{K_{1} K_{2} K_{3} K_{4}\left(\mu+\xi+G^{* *}\right)\left[(1-\epsilon) G^{* *}+\mu\right]},
\end{aligned}
$$

with,

$$
\begin{aligned}
A^{*} & =(1-\epsilon)\left[(1-p)\left(\psi_{2} \sigma_{u} K_{3}+\psi_{1} \sigma_{e} K_{2}\right)+K_{1} K_{2} \sigma_{e} p\right], \\
B^{*} & =\sigma_{e} K_{1} K_{2}(1-\epsilon)(p \mu+\xi)+\mu(1-p)\left(\sigma_{e} K_{2} \psi_{1}+\sigma_{u} K_{3} \psi_{2}\right), \\
C^{*} & =\left[K_{1} p+\psi_{1}(1-p)\right](1-\epsilon), \\
D^{*} & =K_{1}(1-\epsilon)(\xi+p \mu)+\psi_{1} \mu(1-p) .
\end{aligned}
$$

Substituting (12) into (11), and simplifying, leads to $G^{* *}=0$ (corresponding to the DFE, $\mathcal{X}$ ) and the following quadratic equation (in terms of $G^{* *}$ ):

$$
a_{11}^{*}\left(G^{* *}\right)^{2}+a_{12}^{*} G^{* *}+a_{13}^{*}=0,
$$

where,

$$
\begin{aligned}
a_{11}^{*}= & K_{3} K_{4}(1-\epsilon)(1-p)\left(K_{2}+\sigma_{u}\right)+C^{*}+A^{*}, \\
a_{12}^{*}= & K_{1} K_{2} K_{3} K_{4}[(1-p)(1-\epsilon)+p]+\mu K_{3} K_{4}(1-p)\left(K_{2}+\sigma_{u}\right)+K_{2} K_{4} D^{*}+B^{*} \\
& -\beta\left[K_{3} K_{4}(1-p)(1-\epsilon)\left(K_{2}+\sigma_{u} \eta_{u}\right)+(1-\kappa)\left(K_{2} K_{4} C^{*}+\eta_{e} A^{*}\right)\right], \\
a_{13}^{*}= & K_{1} K_{2} K_{3} K_{4}(\mu+\xi)\left(1-\mathcal{R}_{e f f}\right) .
\end{aligned}
$$


Thus, the following results from the quadratic equation (13).

Theorem 3. (a) If $a_{12}^{*}>0$ then model (1) has forward bifurcation at $\mathcal{R}_{\text {eff }}=1$.

(b) If $a_{12}^{*}<0$, then the model (1) undergoes backward bifurcation at $\mathcal{R}_{\text {eff }}=1$.

Theorem 4. (a) If $a_{12}^{*}>0$ and

(i) $a_{13}^{*} \geq 0$, the model (1) has no positive equilibrium

(ii) $a_{13}^{*}<0$, the model (1) has a unique positive equilibrium

(b) If $a_{12}^{*}<0$ and $a_{13}^{*}>0$ and

(i) $\left(a_{12}^{*}\right)^{2}-4 a_{11}^{*} a_{13}^{*}>0$, the model (1) has two positive equilibria,

(ii) $\left(a_{12}^{*}\right)^{2}-4 a_{11}^{*} a_{13}^{*}=0$, the model (1) has a unique positive equilibrium,

(iii) $\left(a_{12}^{*}\right)^{2}-4 a_{11}^{*} a_{13}^{*}<0$, the model (1) has no positive equilibrium.

(c) If $a_{12}^{*}<0$ and $a_{13}^{*} \leq 0$, the model (1) has a unique positive equilibrium.

Since all the model parameters are non-negative (and $0<\epsilon<1,0<\kappa<1$ ), it is clear that $a_{11}^{*}>0$. We consider the following cases:

Case I. Suppose $\mathcal{R}_{\text {eff }}>1$. Then, clearly $a_{13}^{*}<0$. Thus, the quadratic equation (11) is concave up and has two real roots of opposite signs. This implies that the model has a unique positive equilibrium whenever $\mathcal{R}_{\text {eff }}>1$.

Case II. Suppose $\mathcal{R}_{\text {eff }}=1$. Then $a_{13}^{*}=0$ and the quadratic reduces to $G^{* *}\left(a_{11}^{*} G^{* *}+\right.$ $\left.a_{12}^{*}\right)=0$, with roots $G^{* *}=0$ (corresponding to the disease-free equilibrium, $\mathcal{X}$ ) and $G^{* *}=\frac{-a_{12}^{*}}{a_{11}^{*}}$. Thus, for $\mathcal{R}_{e f f}=1$, the model has a unique positive endemic equilibrium when $a_{12}^{*}<0$.

Case III. Suppose $\mathcal{R}_{\text {eff }}<1$. Then $a_{13}^{*}>0$ and equation (13) has either zero, one or two positive real roots. In order to obtain two positive real roots we need $\left(a_{12}^{*}\right)^{2}-4 a_{11}^{*} a_{13}^{*}>0$ and $a_{12}^{*}<0$. If $a_{12}^{*}<0$ and $\left(a_{12}^{*}\right)^{2}-4 a_{11}^{*} a_{13}^{*}=0$, then 
there is one positive real root. Otherwise, there is no positive solution. This case indicates the possibility of a backward bifurcation in the model (1) when $\mathcal{R}_{\text {eff }}<1$ (since it suggests the possibility of multiple endemic equilibria when $\left.\mathcal{R}_{\text {eff }}<1\right)$.

It should be noted that Theorem 3 does not give a local description of the bifurcating curve including its stability. Thus, it is instructive to determine the local behaviour of the bifurcating branch. Therefore, we alternatively use centre manifold theorem, in line with Castillo-Chavez \& Song (2004), to prove the existence of backward bifurcation. The proof of the following theorem is given in Appendix.

\section{Theorem 5 .}

If (20) holds, then the model (1) has a backward bifurcation at $\mathcal{R}_{\text {eff }}=1$ and the bifurcating branch is unstable near $\mathcal{R}_{\text {eff }}=1$.

To illustrate this phenomenon with respect to the above Theorem, the same parameter values for Figure 4B are used and the backward bifurcation diagrams are depicted in Figure 8. For this set of parameter values, the associated backward bifurcation coefficients $(a$ and $b$ ) have the values: $a=0.02069982715$ and $b=1.930595939$.

It is worth noting that when $\epsilon=1$ (i.e., public health education campaign is $100 \%$ effective), the threshold quantity $\mathcal{R}_{\text {eff }}$ reduces to

$$
\tilde{\mathcal{R}}_{e f f}=\left.\mathcal{R}_{e f f}\right|_{\epsilon=1}=\frac{\beta(B+C)}{K_{1} K_{2} K_{3} K_{4}(\xi+\mu)} .
$$

Similarly, the coefficients of the quadratic (13) reduce to

$$
\begin{aligned}
& a_{11}^{*}=0, \\
& a_{12}^{*}=K_{1} K_{2} K_{3} K_{4} p+\mu(1-p)\left[K_{3} K_{4}\left(K_{2}+\sigma_{u}\right)+K_{2} \psi_{1}\left(K_{4}+\sigma_{e}\right)+\sigma_{u} K_{3} \psi_{2}\right]>0, \\
& a_{13}^{*}=K_{1} K_{2} K_{3} K_{4}(\mu+\xi)\left(1-\tilde{\mathcal{R}}_{e f f}\right) .
\end{aligned}
$$


Thus, the quadratic equation (13) becomes linear in $G^{* *}$, with $G^{* *}=\frac{-a_{13}^{*}}{a_{12}^{*}}$. In this case, the model (1) has a unique endemic equilibrium if and only if $\tilde{\mathcal{R}}_{\text {eff }}>1$ (i.e., $a_{13}^{*}<0$ ) and no endemic equilibria when $\tilde{\mathcal{R}}_{e f f}<1$ (since, in this case, $G^{* *}=\frac{-a_{13}^{*}}{a_{12}^{*}}<0$ ). Hence, backward bifurcation is ruled out in this case (since no multiple endemic equilibria exist when $\left.\tilde{\mathcal{R}}_{e f f}<1\right)$. Alternatively, it can easily be seen that the inequality (20) fails whenever $\epsilon=1$. This result is summarized below.

\section{Theorem 6 .}

The model (1) with $\epsilon=1$ does not have a positive endemic equilibrium when $\tilde{\mathcal{R}}_{\text {eff }}<1$.

Further, to show that HIV elimination is independent of the initial sizes of the sub-populations of the model when $\epsilon=1$ (i.e., the efficacy of public health education is $100 \%$ ), we claim the following result:

Theorem 7. The DFE of the model (1) with $\epsilon=1$ is GAS in $\mathcal{D}$ if $\tilde{\mathcal{R}}_{\text {eff }} \leq \frac{S_{u}^{*}}{N^{*}} \leq 1$.

Proof. Consider the model (1) with $\epsilon=1$. Further, consider the Lyapunov function

$$
\mathcal{F}=f_{1} I_{u}+f_{2} A_{u}+f_{3} I_{e}+f_{4} A_{e}
$$

where,

$$
\begin{aligned}
f_{1} & =(1-\kappa)\left[\psi_{1} K_{2} K_{4}+\eta_{e} \psi_{2} \sigma_{u} K_{3}+\eta_{e} \sigma_{e} \psi_{1} K_{2}\right]+K_{3} K_{4}\left(K_{2}+\eta_{u} \sigma_{u}\right) \\
f_{2} & =K_{1} K_{3}\left[\eta_{u} K_{4}+\eta_{e} \psi_{2}(1-\kappa)\right] \\
f_{3} & =K_{1} K_{2}(1-\kappa)\left[K_{4}+\eta_{e} \sigma_{e}\right] \\
f_{4} & =K_{1} K_{2} K_{3} \eta_{e}(1-\kappa)
\end{aligned}
$$

with Lyapunov derivative given by (where a dot represents differentiation with respect to $t$ ) 


$$
\begin{aligned}
\dot{\mathcal{F}} & =f_{1} \dot{I}_{u}+f_{2} \dot{A}_{u}+f_{3} \dot{I}_{e}+f_{4} \dot{A}_{e} \\
& =f_{1}\left[\lambda_{u} S_{u}+(1-\kappa) \lambda_{e} S_{u}-K_{1} I_{u}\right]+f_{2}\left(\sigma_{u} I_{u}-K_{2} A_{u}\right) \\
& +f_{3}\left(\psi_{1} I_{u}-K_{3} I_{e}\right)+f_{4}\left(\sigma_{e} I_{e}+\psi_{2} A_{u}-K_{4} A_{e}\right), \\
& =K_{1} K_{2} K_{3} K_{4}\left(\frac{N^{*} S_{u}}{S_{u}^{*} N} \tilde{\mathcal{R}}_{e f f}-1\right) I_{u}+K_{1} K_{2} K_{3} K_{4} \eta_{u}\left(\frac{N^{*} S_{u}}{S_{u}^{*} N} \tilde{\mathcal{R}}_{e f f}-1\right) A_{u} \\
& +K_{1} K_{2} K_{3} K_{4}\left(\frac{N^{*} S_{u}}{S_{u}^{*} N} \tilde{\mathcal{R}}_{e f f}-1\right) I_{e}+K_{1} K_{2} K_{3} K_{4} \eta_{e}(1-\kappa)\left(\frac{N^{*} S_{u}}{S_{u}^{*} N} \tilde{\mathcal{R}}_{e f f}-1\right) A_{e} \\
& -I_{u}\left[K_{1}(1-\kappa)\left(\psi_{1} K_{2} K_{4}+\eta_{e} \sigma_{e} \psi_{1} K_{2}\right)\right] \\
& =K_{1} K_{2} K_{3} K_{4}\left(I_{u}+\eta_{u} A_{u}+I_{e}+\eta_{e}(1-\kappa) A_{e}\right)\left(\frac{N^{*} S_{u}}{S_{u}^{*} N} \tilde{\mathcal{R}}_{e f f}-1\right) \\
& -I_{u}\left[K_{1}(1-\kappa)\left(\psi_{1} K_{2} K_{4}+\eta_{e} \sigma_{e} \psi_{1} K_{2}\right)\right] \\
& \leq K_{1} K_{2} K_{3} K_{4}\left(I_{u}+\eta_{u} A_{u}+I_{e}+\eta_{e}(1-\kappa) A_{e}\right)\left(\frac{N^{*}}{S_{u}^{*}} \tilde{\mathcal{R}}_{e f f}-1\right) \\
& -I_{u}\left[K_{1}(1-\kappa)\left(\psi_{1} K_{2} K_{4}+\eta_{e} \sigma_{e} \psi_{1} K_{2}\right)\right] \quad \text { since } \quad S_{u} \leq N \quad \text { in } \mathcal{D} \\
& \leq 0 \quad \text { for } \quad \tilde{\mathcal{R}}_{e f f} \leq \frac{S_{u}^{*}}{N^{*}} \leq 1 .
\end{aligned}
$$

Thus, $\dot{\mathcal{F}} \leq 0$ if $\tilde{\mathcal{R}}_{e f f} \leq \frac{S_{u}^{*}}{N^{*}}$ with $\dot{\mathcal{F}}=0$ if and only if $I_{u}=A_{u}=I_{e}=A_{e}=0$. Further, the largest compact invariant set in $\left\{\mathcal{X}:\left(S_{u}^{*}, S_{e}^{*}, I_{u}^{*}, A_{u}^{*}, I_{e}^{*}, A_{e}^{*}\right) \in \mathcal{D}\right.$ : $\dot{\mathcal{F}}=0\}$ is the singleton $\mathcal{D}_{\mathcal{X}}$. It follows from the LaSalle Invariance Principle (LaSalle, 1976), that every solution to the equations in (1) with initial conditions in $\mathcal{D}$ converge to $\mathcal{D}_{\mathcal{X}}$ as $t \rightarrow \infty$. That is, the disease dies out. Further, substituting $I_{u}=A_{u}=I_{e}=A_{e}=0$ in the model shows that $S_{u} \rightarrow S_{u}^{*}$ and $S_{e} \rightarrow S_{e}^{*}$ as $t \rightarrow \infty$. Thus, $\left(S_{u}, S_{e}, I_{u}, A_{u}, I_{e}, A_{e}\right) \rightarrow\left(S_{u}^{*}, S_{e}^{*}, 0,0,0,0\right)$ as $t \rightarrow \infty$. Hence, since the region $\mathcal{D}$ is positively-invariant, it follows that the DFE of (1), with $\epsilon=1$, is GAS in $\mathcal{D}$ for all non-negative initial conditions, whenever $\tilde{\mathcal{R}}_{\text {eff }} \leq \frac{S_{u}^{*}}{N^{*}} \leq 1$.

In summary, it is clear from Theorems 6 and 7 that that the backward bifurcation phenomenon of the model is caused by the imperfect nature of the public health education campaign (i.e., $0<\epsilon<1$ ). In the case where the 
public health education is perfect, $\tilde{\mathcal{R}}_{e f f} \leq \frac{S_{u}^{*}}{N^{*}} \leq 1$ is necessary and sufficient condition for the effective control of HIV in the community. In other words, the public health education with perfect efficacy could lead to effective control (or theoretical elimination) of HIV in the community provided the associated threshold quantity, $\tilde{\mathcal{R}}_{e f f}$, is brought to (and maintained at) a value less than $\frac{S_{u}^{*}}{N^{*}}$. Thus, this study emphasizes the pressing need for the design of perfect public health education campaign to handle HIV.

Theorem 8. The DFE of the model (1) with $\epsilon=1$ does not undergo backward bifurcation at $\tilde{\mathcal{R}}_{\text {eff }}=1$.

Proof. The result follows from Theorem 6 , where the model has no positive equilibrium when $\tilde{\mathcal{R}}_{e f f}<1$, and Theorem 7 , where the DFE of the model (1) is GAS in $\mathcal{D}$ if $\tilde{\mathcal{R}}_{e f f} \leq \frac{S_{u}^{*}}{N^{*}} \leq 1$.

\section{Conclusions}

A realistic deterministic model, which incorporates public health education campaign as a sole intervention strategy for HIV/AIDS prevention, is designed and rigorously analyzed to get insight into its dynamical features and to obtain associated epidemiological thresholds. Some of the main theoretical findings of the study are:

- Under certain conditions, the model (1) undergoes backward bifurcation, when the reproduction number $\left(\mathcal{R}_{\text {eff }}\right)$ is less than unity. The backward bifurcation phenomenon resulted from the imperfect nature of the public health education program.

- For the case when the public health education program is $100 \%$ effective, the disease-free equilibrium of the model (1) is globally-asymptotically stable when- 
ever the associated reproduction number is less than or equal to a quantity less than unity.

- Threshold analysis of the effective reproduction number shows that the use of public health education campaign could have positive, no, or detrimental impact depending on whether or not an impact factor, defined as $\Upsilon$, is less than, equal to, or greater than unity (this result is also expressed in terms of a measure of risky behaviour, denoted by $\nabla$, given by (6)).

The impact of public health education strategies are assessed numerically by simulating the model with a reasonable set of parameter values (mostly chosen from the literature) and initial (demographic) data from five different countries (India, Nigeria, China, Ethiopia, and Russia) where the number of HIV-infected people is expected to grow. Numerical simulations of the model show the following:

- The universal use of public heath education campaign in India, Nigeria, China, Ethiopia, and Russia could avert more than 1.1590 million, 0.7580 million, 0.3858 million, 0.5731 million, and 0.253 million new HIV cases within a year, respectively.

- The universal strategy is more effective than any other strategy in reducing new HIV cases.

- Combining Strategies I, III and IV is the next most effective in reducing the total number of new cases (after the universal strategy).

- Amongst the 2-group combined strategies, combining Strategies I and IV is most effective than some 3-group combined strategies. 
- Strategy I averts more new cases in comparison to all other single-group strategies (and some 3-group combination of strategies).

- The prospect of effective control of HIV increases with increasing efficacy and coverage rate of the public health education campaign.

Overall, this study shows that an effective public health education campaign which focuses on change of risky behaviour with a reasonable coverage level could help in stemming HIV/AIDS in the countries studied. This requires a concerted effort from all the stake holders especially the governments of the respective countries.

\section{Acknowledgments}

$\mathrm{NH}$ acknowledges, with thanks, the support of Kano State Government of Nigeria. ABG acknowledges the support, in part, of the Natural Science and Engineering Research Council (NSERC) and Mathematics of Information Technology and Complex Systems (MITACS) of Canada. The authors are grateful to C.N. Podder and S.M. Garba for their comments. The authors are grateful to the anonymous reviewers for their constructive comments, which have improved the manuscript. 


\section{Appendix: Proof of Theorem 5}

$$
\begin{aligned}
& \frac{d x_{1}}{d t}=\phi_{1}=\Pi(1-p)-(\xi+\mu) x_{1}-\frac{\beta x_{1}\left[\left(x_{3}+\eta_{u} x_{4}\right)+(1-\kappa)\left(x_{5}+\eta_{e} x_{6}\right)\right]}{x_{1}+x_{2}+x_{3}+x_{4}+x_{5}+x_{6}}, \\
& \frac{d x_{2}}{d t}=\phi_{2}=\Pi p+\xi x_{1}-\frac{\beta(1-\epsilon) x_{2}\left[\left(x_{3}+\eta_{u} x_{4}\right)+(1-\kappa)\left(x_{5}+\eta_{e} x_{6}\right)\right]}{x_{1}+x_{2}+x_{3}+x_{4}+x_{5}+x_{6}}-\mu x_{2}, \\
& \frac{d x_{3}}{d t}=\phi_{3}=\frac{\beta x_{1}\left[\left(x_{3}+\eta_{u} x_{4}\right)+(1-\kappa)\left(x_{5}+\eta_{e} x_{6}\right)\right]}{x_{1}+x_{2}+x_{3}+x_{4}+x_{5}+x_{6}}-K_{1} x_{3} \\
& \frac{d x_{4}}{d t}=\phi_{4}=\sigma_{u} x_{3}-K_{2} x_{4} \\
& \frac{d x_{5}}{d t}=\phi_{5}=\frac{\beta(1-\epsilon) x_{2}\left[\left(x_{3}+\eta_{u} x_{4}\right)+(1-\kappa)\left(x_{5}+\eta_{e} x_{6}\right)\right]}{x_{1}+x_{2}+x_{3}+x_{4}+x_{5}+x_{6}}+\psi_{1} x_{3}-K_{3} x_{5}, \\
& \frac{d x_{6}}{d t}=\phi_{6}=\sigma_{e} x_{5}+\psi_{2} x_{4}-K_{4} x_{6} .
\end{aligned}
$$

514 The Jacobian of $\Phi=\left(\phi_{1}, \phi_{2}, \phi_{3}, \phi_{4}, \phi_{5}, \phi_{6}\right)^{T}$, around the DFE $\mathcal{X}$, denoted by $J_{\beta}$, is 515 given by

$$
J_{\beta}=\left(\begin{array}{cccccc}
-\xi-\mu & 0 & -\beta H_{1} & -\beta \eta_{u} H_{1} & -\beta(1-\kappa) H_{1} & -\beta \eta_{e}(1-\kappa) H_{1} \\
\xi & -\mu & -\beta H_{2} & -\beta \eta_{u} H_{2} & -\beta(1-\kappa) H_{2} & -\beta \eta_{e}(1-\kappa) H_{2} \\
0 & 0 & \beta H_{1}-K_{1} & \beta \eta_{u} H_{1} & \beta(1-\kappa) H_{1} & \beta \eta_{e}(1-\kappa) H_{1} \\
0 & 0 & \sigma_{u} & -K_{2} & 0 & 0 \\
0 & 0 & \beta H_{2}+\psi_{1} & \beta \eta_{u} H_{2} & \beta(1-\kappa) H_{2}-K_{3} & \beta \eta_{e}(1-\kappa) H_{2} \\
0 & 0 & 0 & \psi_{2} & \sigma_{e} & -K_{4}
\end{array}\right),
$$


517 (4), that

where, $H_{1}=\frac{\mu(1-p)}{\xi+\mu}$ and $H_{2}=\frac{(1-\epsilon)(p \mu+\xi)}{\xi+\mu}$. It can also be shown from $J_{\beta}$, as in

$$
R_{e f f}=\frac{\beta(A+B+C)}{K_{1} K_{2} K_{3} K_{4}(\xi+\mu)}
$$

Consider the case when $\mathcal{R}_{\text {eff }}=1$ and $\beta$ is chosen as a bifurcation parameter. Solving (17) for $\mathcal{R}_{\text {eff }}=1$ gives

$$
\beta=\beta^{* *}=\frac{K_{1} K_{2} K_{3} K_{4}(\xi+\mu)}{A+B+C}
$$

Note that the above linearized system, of the transformed system (16) with $\beta=\beta^{* *}$, has a zero eigenvalue. Hence, the center manifold theory Carr (1981) can be used to analyze the dynamics of (16) near $\beta=\beta^{* *}$.

521

\section{Eigenvectors of $\left.J_{\beta}\right|_{\beta=\beta^{* *}}$ :}

The right and left eigenvectors associated with the zero eigenvalue of the Jacobian $J_{\beta}$ evaluated at $\beta^{* *}$ are given, respectively, by $\mathbf{w}=\left[w_{1}, w_{2}, w_{3}, w_{4}, w_{5}, w_{6}\right]^{T}$ and $\mathbf{v}=$ $\left[v_{1}, v_{2}, v_{3}, v_{4}, v_{5}, v_{6}\right]$, where

$$
\begin{aligned}
& w_{1}=-\frac{\beta^{* *} H_{1}\left\{w_{3}+\eta_{u} w_{4}+(1-\kappa) w_{5}+\eta_{e}(1-\kappa) w_{6}\right\}}{\xi+\mu}<0, \\
& w_{2}=\frac{\xi w_{1}-\beta^{* *} H_{2}\left\{w_{3}+\eta_{u} w_{4}+(1-\kappa) w_{5}+\eta_{e}(1-\kappa) w_{6}\right\}}{\mu}<0, \\
& w_{3}=w_{3}>0, \quad w_{4}=\frac{\sigma_{u}}{K_{2}} w_{3}, \\
& w_{5}=w_{5}>0, \quad w_{6}=\frac{\psi_{2} w_{4}+\sigma_{e} w_{5}}{K_{4}}, \\
& v_{1}=v_{2}=0, \quad v_{3}=v_{3}>0, \quad v_{4}=\frac{\beta^{* *} \eta_{u} H_{1} v_{3}+\beta^{* *} \eta_{u} H_{2} v_{5}+\psi_{2} v_{6}}{K_{2}}, \\
& v_{5}=v_{5}>0, \quad v_{6}=\frac{\beta^{* *} \eta_{e}(1-\kappa)\left(H_{1} v_{3}+H_{2} v_{5}\right)}{K_{4}} .
\end{aligned}
$$


To determine the direction of bifurcation, following Castillo-Chavez \& Song (2004), we find the signs of $a$ and $b$, where

$$
a=\sum_{k, i, j=1}^{6} v_{k} w_{i} w_{j} \frac{\partial^{2} \phi_{k}}{\partial x_{i} \partial x_{j}}(0,0) \quad \text { and } \quad b=\sum_{k, i=1}^{6} v_{k} w_{i} \frac{\partial^{2} \phi_{k}}{\partial x_{i} \partial \beta^{* *}}(0,0) .
$$

Hence, $a>0$ iff

$$
P_{12}>P_{13}
$$

For the sign of $\mathbf{b}$, we substitute vectors $\mathbf{v}$ and $\mathbf{w}$ and the respective associated nonzero partial derivatives of $\Phi$ at the DFE into

$$
b=\sum_{k, i=1}^{6} v_{k} w_{i} \frac{\partial^{2} \phi_{k}}{\partial x_{i} \partial \beta^{* *}}(0,0)
$$

which gives,

$$
b=\frac{(1-\epsilon)(p \mu+\xi) v_{5}+v_{3} \mu(1-p)}{\xi+\mu} P_{11}>0
$$




\section{REFERENCES}

ANDERSON, R. M.(1988) The role of mathematical models in the study of HIV transmission and the epidemiology of AIDS. J. Acquir. Immune Defic. Syndr., 1, 241-256.

ANDERSON, R.M. \& MAY, R. M.(1991) Infectious Diseases of Humans. Oxford: Oxford Univ. Press, pp. 17-19.

BERKER, M.H. \& JOSEPH, J.G.(1998) AIDS and behavioral change to reduce risk: A review. American Journal of public health, 78, 394-410.

BLOWER, S.M. \& DOWLATABADI, H.(1994) Sensitivity and Uncertainty Analysis of Complex Models of Disease Transmission. Internat. Stat. Rev., 62, 229-243.

BLOWER, S.M. \& MCLEAN, A.R.(1994) Prophylactic vaccines, risk behaviour change, and the probability of eradicating HIV in San Francisco. Science, 265, 4511454

BORTOlotti, F., STIVANEllo, A., NOVENTA, F., FORZA, G., PAVANELlO, N. \& BERTOLINI, A.(1992) Sustained AIDS education campaigns and behavioural changes in Italian drugs abusers. Eur. J. Epidemiol., 8, 264-267.

CARR J. (1981) Applications Centre Manifold Theory. New York: Springer-Verlag.

CASTILlO-CHAVEZ, C. \& SONG, B.(2004) Dynamical models of tuberculosis and their applications. Math. Biosci. Eng., 1, 361-404. 
CASSELl, M.M., HALPERIN, D.T., SHELTON, J.D. \& STANTON, D.(2006) Risk

KEnDALL, M.G., \& STUART, A. (1979) The Advanced Theory of Statistics, Vol. 2: Inference and Relationship. London:Charles Griffin, pp. 82-108.

DANIEL, L. \& RAND, S.L.(2003) Behaviour and communication change in reducing HIV: is Uganda unique? African Journal of AIDS Research, 2, 9-21.

DEL VALlE, S., ECANGELISTA, A.M., VELASCO, M.C., KRIBS-ZALETA, C.M. \& HSU SCHMITZ, S.-F.(2004) Effects of education, vaccination and treatment on HIV transmission in homosexuals with genetic heterogeneity. Math. Biosci., 187, 111133.

DE WALQUE, D.(2007) How does the impact of an HIV/AIDS information campaign vary with educational attainment? Evidence from rural Uganda. Journal of Dev. Eco. 84, 686-714.

ELBASHA, E.H. \& GUMEL, A.B.(2006) Theoretical assessment of public health impact of imperfect prophylactic HIV-1 vaccines with therapeutic benefits. Bull. Math. Biol., 68, 577-614.

FYLKESNES, K., MUSONDA, R.M., SICHONE, M., NDHLOVU, Z., TEMBO, F. \& MONZE, M.(2001) Declining HIV prevalence and risk behaviours in Zambia: evidence 
from surveillance and population-based surveys. AIDS, 15, 907-916.

GARBA S.M. \& GUMEL A.B. (2010) Mathematical Recipe for HIV Elimination in Nigeria, Journal of Nig. Math. Soc., to appear.

GREEN, E.C., HALPERIN, D.T., NANTULYA, V. \& HOGLE, J.A.(2006) Ugandas HIV prevention success: the role of sexual behavior change and the national response. AIDS Behav., 10, 335-346.

GUMEL, A.B., MCCLUSKEY, C. C. \& VAN DEN DRIESSCHE, P.(2006) Mathematical study of a staged-progression HIV model with imperfect vaccine. Bull. Math. Biol., 68, 2105-2128.

HADELER, K.P. \& VAN DEN DRIESSCHE, P. (1997) Backward bifurcation in epidemic control. Math. Biosci. 146, 15-35.

HALE, J.K. (1969) Ordinary Differential Equations. New York: John Wiley and Sons.

HETHCOTE, H.W.(2000) The mathematics of infectious diseases. SIAM Rev., 42 599-653.

HYMAN, J. M., LI, J. \& STANLEY, E.A.(1999) The differential infectivity and staged progression models for the transmission of HIV. Math. Biosci., 208, 227-249.

KAREN, R.D. \& SUSAN, C.W.(1999). The effectiveness of condoms in reducing heterosexual transmission of HIV. Family Planning Perspectives, 31, 272-279. 
KEITSHOKIL, M. D., NAOMI, S., MARIE, B. S., MIRIAM, N. \& MOTSHEDISI, S.(2007) HIV/AIDS Education, Prevention and Control Course (BNS101): The Way Forward. JANAC, 18, 22-31.

KRIBS-ZALETA, C. \& VALESCO-HERNANDEZ, J.(2000) A simple vaccination model with multiple endemic states. Math. Biosci., 164, 183-201.

LAKSHMIKANTHAM, V. LEELA, S. \& MARTYNYUK, A. A. (1989). Stability Analysis of Nonlinear Systems. Marcel Dekker, Inc., New York and Basel, pp. 155170.

LASALLE J.P. (1976) The Stability of Dynamical Systems. Regional Conference Series in Applied Mathematics, SIAM, Philadelphia.

Lindan, C., ALlen, S., CARAEL, M., NSENGUMUREMyi, F., DE PERRE, P. V., SERUfiliRA, A., TICE, J., BLACK, D., THOMAS, C. \& HULlEY, S. (1991) Knowledge, Attitudes, and Percieved Risk of AIDS among Urban Rwandan WomenRelationship to HIV-Infection and Behaviour Change. AIDS, 5, 993-1002.

MORTON, M., NELSON, L., WALSH, C., ZIMMERMAN, S. \& COE, R. M.(1996) Evaluation of a HIV/AIDS education program for adolescents. J. Community Health, 21, 23-35.

MUKANDAVIRE, Z. \& GARIRA, W.(2007) Effects of public health educational campaigns and the role of sex workers on the spread of HIV/AIDS among heterosexu- 
als. Theor. Popul. Biol. 72, 346-365.

MUKANDAVIRE, Z., GARIRA, W. \& TCHUENCHE, J. M. (2009) Modelling effects of public health educational campaigns on HIV/AIDS transmission dynamics. Applied Mathematical Modelling 33, 20842095.

National Intelligence Council(2002) The Next Wave of HIV/AIDS: Nigeria, Ethiopia, Russia, India, and China. www.fas.org/irp/nic/hiv-aids.html accessed 26 September 2009 .

PÉREZ V. R., BARRAlES C. I., JARA P. J., PALMA R. V. \& CEBAllos M. A.(2008) Knowledge of HIV/AIDS among adolescents in Chillán Chile. Midwifery, 24, 503-508.

SHAROMI O. \& Gumel. A.B.(2008) Dynamical analysis of a multi-strain model of HIV in the presence of antiretroviral drugs. Journal of Biological Dynamics, 2, 323345.

SMITH, R. J. \& BLOWER, S.M.(2004) Could disease-modifying HIV vaccine cause population-level perversity? Lancet. Infect. Dis., 4, 636-639.

Uganda Bureau of Statistics Census(UBSC)(1991) Population and Social Statistics. http://www.ubos.org/?st=pagerelations2 $i d=17$ p=related 20pages 202:Population, accessed 25 September 2009.

United Nations Department of Economic and Social Affairs/Population Division (2004) World Population Monitoring 2002: Reproductive Rights and Reproductive Health, United Nations, NewYork, http : //www.un.org/esa/population/publications/2003monitoring/WorldPopMonitoring $2002 . p d f$, 
666

685

686

accessed 27 September 2009.

UNAIDS(2007) AIDS epidemic update.

http://data.unaids.org/pub/EPISlides/2007/2007 epiupdate en.pdf, accessed 07 October 2008 .

UNAIDS(2008) Report on the Global HIV/AIDS Epidemic.

http : //www.unaids.org/en/KnowledgeCentre/HIV Data/GlobalReport/2008/2008 ${ }_{\text {lobal }}$ eport.asp, accessed 25 September 2009.

UNAIDS/WHO/Unicef(2008) Epidemiological Fact Sheets on HIV/AIDS and STI:

Core Data on Epidemiology and Response, Uganda.

www.apps.who.int/globalatlas/predenedReports/EFS2008/full/EFS2008-NG.pdf, accessed 25 September 2009.

VAN DEN DRIESSCHE, P. \& WATMOUGH, J.(2002) Reproduction numbers and sub-threshold endemic equilibria for compartmental models of disease transmission. Math. Biosci., 180, 29-48.

VALESCO-HERNANDEZ, J. X. \& HSIEH, Y. H.(1994) Modelling the effect and behavioral change in HIV transmission dynamics. J. Math. Biol., 32, 233-249.

WILSON, D.(2004) Partner reduction and the prevention of HIV/AIDS. B. M. J., 328, 848-849.

World Factbook(2002) https://www.cia.gov/library/publications/the-world-factbook/index.html, 
accessed 26 September 2009.

688

${ }_{689}$ World Factbook(2008) https://www.cia.gov/library/publications/the-world-factbook/index.html, accessed 26 September 2009.

691 


\begin{tabular}{ll}
\hline \hline Variables & Description \\
\hline \hline & Adult population \\
$S_{u}$ & Uneducated susceptible individuals \\
$S_{e}$ & Educated susceptible individuals \\
$I_{u}$ & Uneducated infecteds with no AIDS symptoms \\
$I_{e}$ & Educated infecteds with no AIDS symptoms \\
$A_{u}$ & Uneducated infecteds with AIDS symptoms \\
$A_{e}$ & Educated infecteds with AIDS symptoms \\
$\lambda_{u}$ & Force of infection of uneducated individuals \\
$\lambda_{e}$ & Force of infection of educated individuals \\
\hline \hline & \\
Parameters & Description \\
\hline \hline & \\
$\mu$ & Recruitment rate of susceptibles \\
$\delta_{u}, \delta_{e}$ & Natural mortality rate \\
$p$ & Disease-induced mortality rates \\
$\xi$ & Fraction of educated newly-recruited individuals \\
$\psi_{1}, \psi_{2}$ & Rate of educating susceptibles \\
$\beta$ & Education rates of individuals in $I_{u}$ and $A_{u}$ classes \\
$\eta_{u}, \eta_{e}$ & Effective contact rate \\
$\epsilon$ & Modification parameters \\
$1-\kappa$ & Efficacy of education in preventing infection \\
$\sigma_{u}, \sigma_{e}$ & Reduction in transmissibility of educated individuals \\
\hline \hline
\end{tabular}

Table 1: Description of Variables and Parameters of the Model (1). 


\begin{tabular}{lll}
\hline \hline Parameters & Nominal value & References \\
\hline \hline & & \\
$\delta_{u}, \delta_{e}$ & $0.47,0.04$ & Gumel et al., 2006 \\
$p, \xi$ & $0.5,0.5$ & Assume \\
$\psi_{1}, \psi_{2}$ & $0.5,0.5$ & Assume \\
$\beta$ & 0.4 & Elbasha \& Gumel $(2006)$ \\
$\eta_{u}, \eta_{e}$ & $1.5,1.2$ & Sharomi \& Gumel(2008) \\
$\epsilon$ & 0.8 & Karen \& Susan (1999) \\
$1-\kappa$ & 0.3 & Assumed \\
$\sigma_{u}, \sigma_{e}$ & $2.6,1 / 15$ & Gumel et al., (2006) Hyman et al., (1999); \\
\hline \hline
\end{tabular}

Table 2: Epidemiological Data for Model (1).

\begin{tabular}{lllllll}
\hline \hline $\begin{array}{l}\text { Demographic } \\
\text { Parameters }\end{array}$ & $\begin{array}{l}\text { India } \\
\text { (millions) }\end{array}$ & $\begin{array}{l}\text { Nigeria } \\
\text { (millions) }\end{array}$ & $\begin{array}{l}\text { China } \\
\text { (millions) }\end{array}$ & $\begin{array}{l}\text { Ethiopia } \\
\text { (millions) }\end{array}$ & $\begin{array}{l}\text { Russia } \\
\text { (millions) }\end{array}$ & References \\
\hline \hline$N(0)$ & 1025.1 & 116.9 & 1285 & 64.5 & 144.7 & United Nations $(2004)$ \\
$1 / \mu$ & 64 (years) & 52 (years) & 71 (years) & 53 (years) & 66 (years) & United Nations $(2004)$ \\
$\Pi$ & $1.51 \%$ & $2.54 \%$ & $0.87 \%$ & $2.64 \%$ & $0.33 \%$ & World Factbook $(2002)$ \\
$S_{u}(0)$ & 1010 & 110 & 800 & 60 & 100 & Assumed \\
$S_{e}(0)$ & 10 & 3.3 & 483.75 & 1.5 & 43.84 & Assumed \\
Infecteds & 5.1 & 3.6 & 1.25 & 3 & 0.86 & World Factbook $(2008)$ \\
$I_{u}(0)$ & 3 & 2 & 1 & 2 & 0.7 & Assumed \\
$I_{e}(0)$ & 1 & 1 & 0.1 & 0.4 & 0.1 & Assumed \\
$A_{u}(0)$ & 1 & 0.5 & 0.1 & 0.4 & 0.05 & Assumed \\
$A_{e}(0)$ & 0.1 & 0.1 & 0.05 & 0.2 & 0.01 & Assumed \\
\hline \hline
\end{tabular}

Table 3: 2002 Demographic of Data Used as Initial Conditions. 


\begin{tabular}{llllll}
\hline \hline Education strategy & $\begin{array}{l}\text { India } \\
(\text { millions })\end{array}$ & $\begin{array}{l}\text { Nigeria } \\
(\text { millions })\end{array}$ & $\begin{array}{l}\text { China } \\
(\text { millions })\end{array}$ & $\begin{array}{l}\text { Ethiopia } \\
(\text { millions })\end{array}$ & $\begin{array}{l}\text { Russia } \\
\text { (millions) }\end{array}$ \\
\hline
\end{tabular}

(A)

\begin{tabular}{llllll}
\hline & & & & & \\
Strategy I & 0.8642 & 0.5474 & 0.3321 & 0.4064 & 0.2116 \\
Strategy II & 0.3633 & 0.2108 & 0.2584 & 0.1390 & 0.1510 \\
Strategy III & 0.5266 & 0.3095 & 0.2912 & 0.2321 & 0.1770 \\
Strategy IV & 0.5862 & 0.3718 & 0.2938 & 0.2510 & 0.1805 \\
\hline
\end{tabular}

(B)

\begin{tabular}{llllll}
\hline & & & & & \\
Strategies I and II & 0.8717 & 0.5564 & 0.3331 & 0.4140 & 0.2119 \\
Strategies I and III & 0.9918 & 0.6290 & 0.3588 & 0.4831 & 0.2320 \\
Strategies I and IV & 1.0359 & 0.6760 & 0.3604 & 0.4966 & 0.2344 \\
Strategies II and III & 0.5353 & 0.3200 & 0.2924 & 0.2408 & 0.1773 \\
Strategies II and IV & 0.5946 & 0.3818 & 0.2950 & 0.2595 & 0.1808 \\
Strategies III and IV & 0.7440 & 0.4723 & 0.3250 & 0.3449 & 0.2046 \\
\hline
\end{tabular}

(C)

\begin{tabular}{llllll} 
Strategies I, II and III & 0.9986 & 0.6373 & 0.3597 & 0.4899 & 0.2322 \\
Strategies I, II and IV & 1.0425 & 0.6839 & 0.3613 & 0.5033 & 0.2347 \\
Strategies I, III and IV & 1.1530 & 0.7508 & 0.3850 & 0.5670 & 0.2531 \\
Strategies II, III and IV & 0.7516 & 0.4814 & 0.3260 & 0.3526 & 0.2049 \\
\hline
\end{tabular}

(D)

\begin{tabular}{llllll} 
Universal Strategy & 1.1590 & 0.7580 & 0.3858 & 0.5731 & 0.2534 \\
\hline
\end{tabular}

Table 4: Total new cases averted within a year using (A) Single targeted public health campaign strategy (B) Pair combination of targeted public health campaign strategies (C) Combination of three strategies (D) Universal strategy. Parameters as in Tables 2 and 3 . 


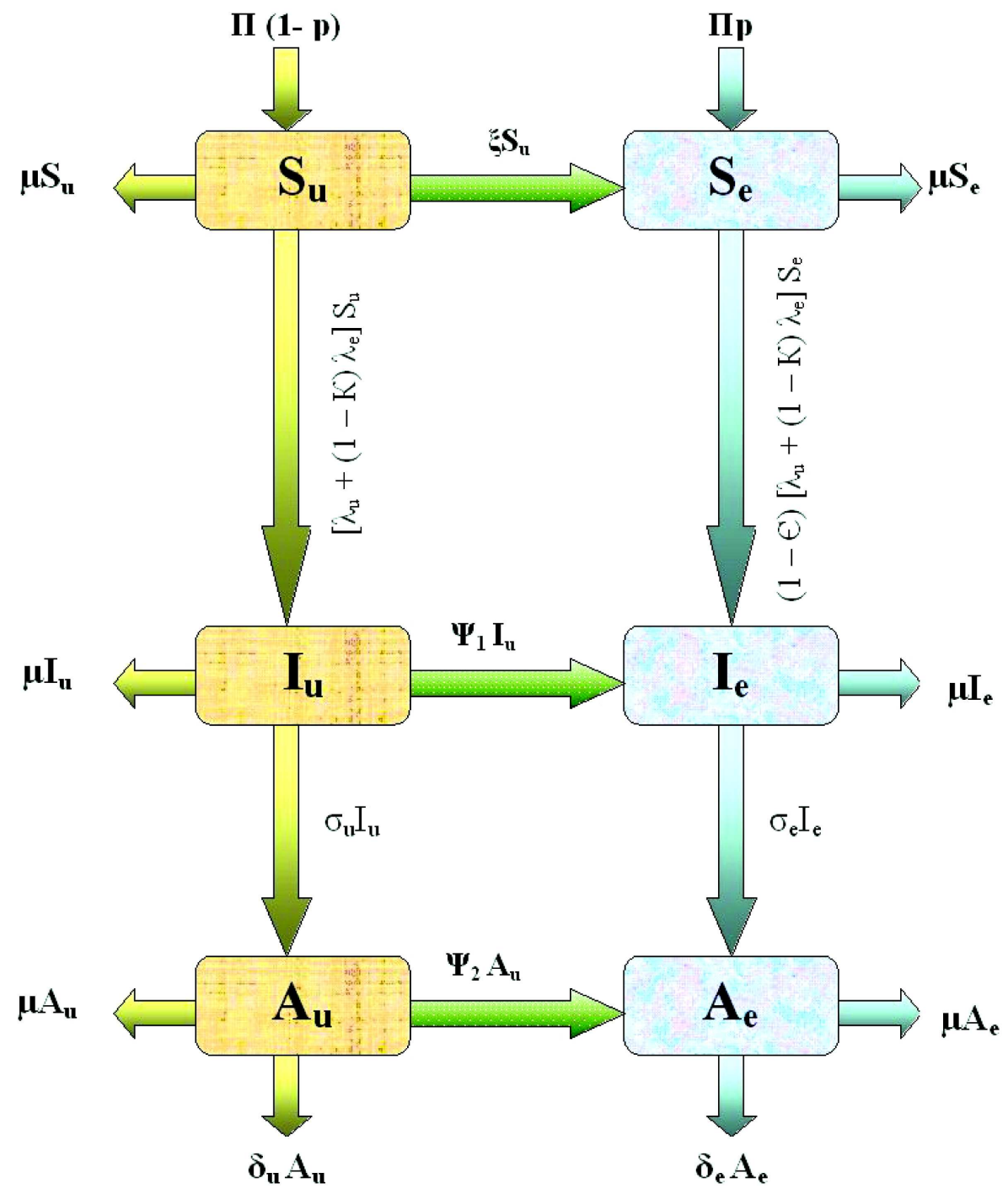

Figure 1: Schematic Diagram of the Model (1) 


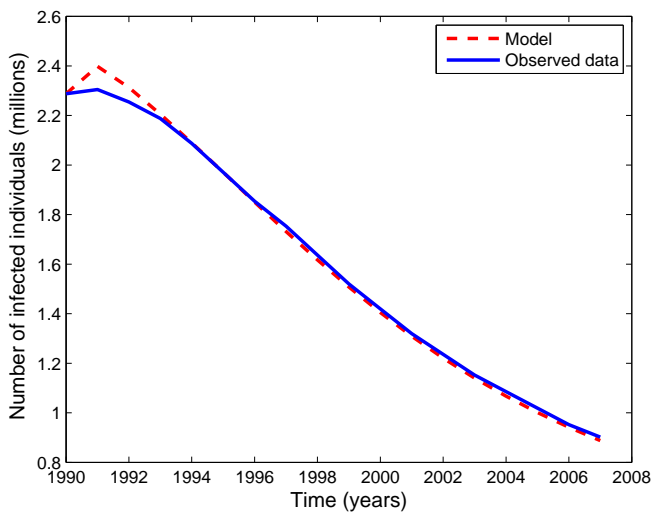

Figure 2: Comparison of observed HIV/AIDS data from Uganda (solid lines) and model prediction (dashed line). Parameter values used are as in Table 2 with $\xi=0.01$, $\psi 1=\psi 2=0.001, \mathrm{p}=0.3$, and $\beta=0.325$.
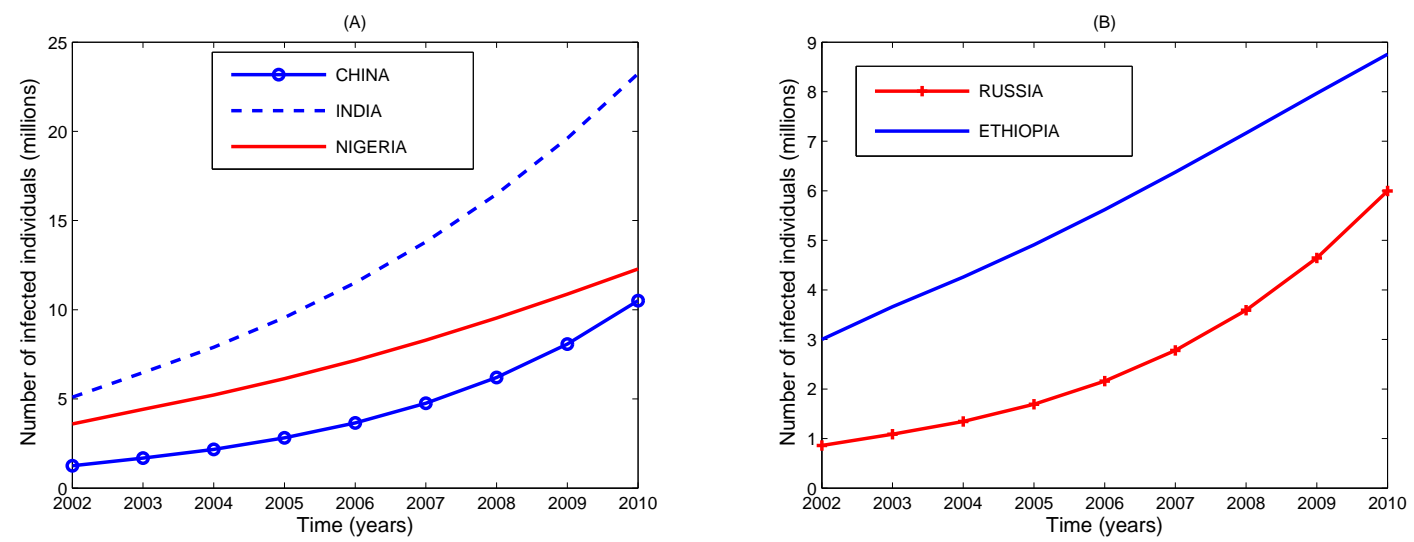

Figure 3: Worst-case scenarios for: (A) China, India and Nigeria; and (B) Russia and Ethiopia. Parameter values used are as in Table 2 with all education-related parameters set to zero. 

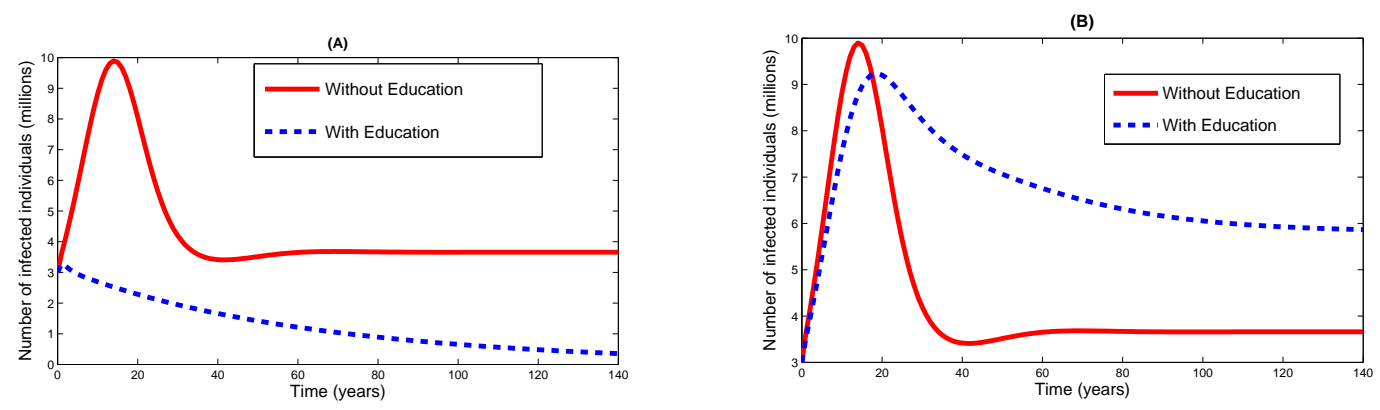

Figure 4: Simulation of the model (1) showing the total infected population as a function of time, using appropriate demographic and epidemiological data for Ethiopia, given in Tables 2 and 3. Dashed line represents the model with public health education campaign and solid line represents the model without education public health education campaign (i.e., all education parameters are zero). For: (A) $\nabla=0.0517<1, \mathcal{R}_{\text {eff }}=$ 0.6898 and $\mathcal{R}_{0 e}=0.6619<\mathcal{R}_{0}=1.3712$; and (B) $\nabla=1.4211>1, \mathcal{R}_{\text {eff }}=1.5866$ and $\mathcal{R}_{0 e}=1.9857>\mathcal{R}_{0}=1.3712$, with $\xi=0.01, p=\psi_{1}=\psi_{2}=0.001$ and $\epsilon=0.4$.

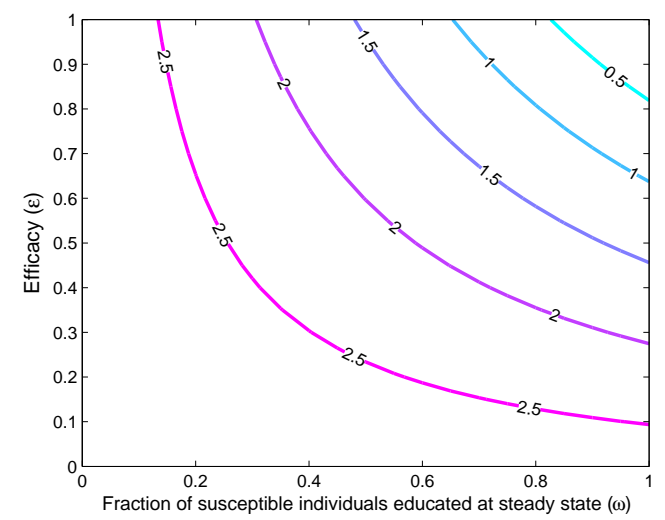

Figure 5: Contour plot of $\mathcal{R}_{\text {eff }}$ as a function of the fraction individuals educated at DFE $(\omega)$ and education efficacy $(\epsilon)$. Parameter values used are as in Table 2. 

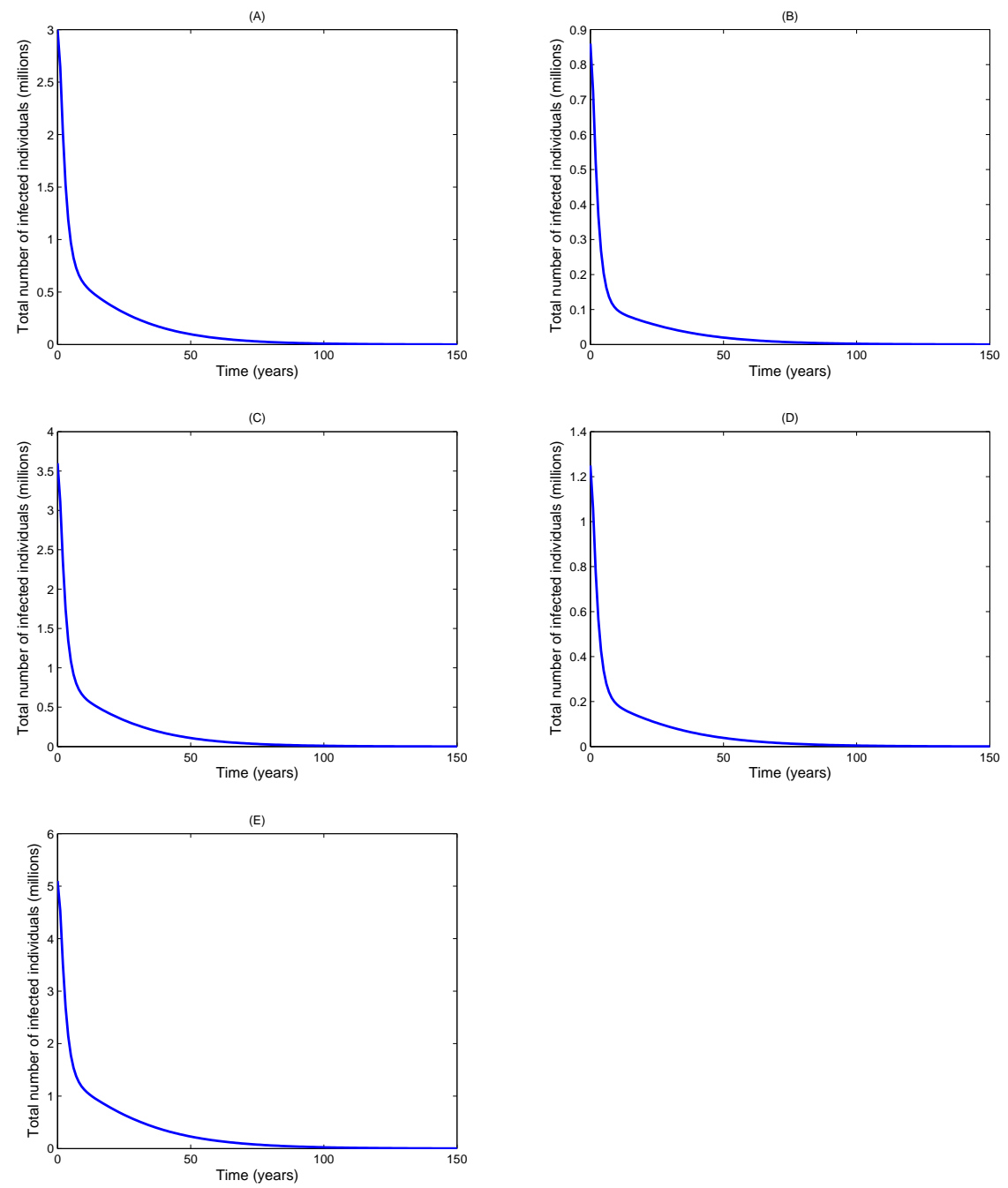

Figure 6: Simulations of the model (1) showing the time needed to eliminate HIV in (A) Ethiopia (B) Russia (C) Nigeria (D) China and (E) India. Parameter values used are as in Tables 2 and 3 with $\xi=p=\epsilon=0.9, \psi_{1}=\psi_{2}=0, \kappa=0.8$ and $\beta=0.2$ (so that, $\nabla=0.1609<1, \mathcal{R}_{e f f}=0.1115$ and $\left.\mathcal{R}_{0 e}=0.1103<\mathcal{R}_{0}=0.6856\right)$. 

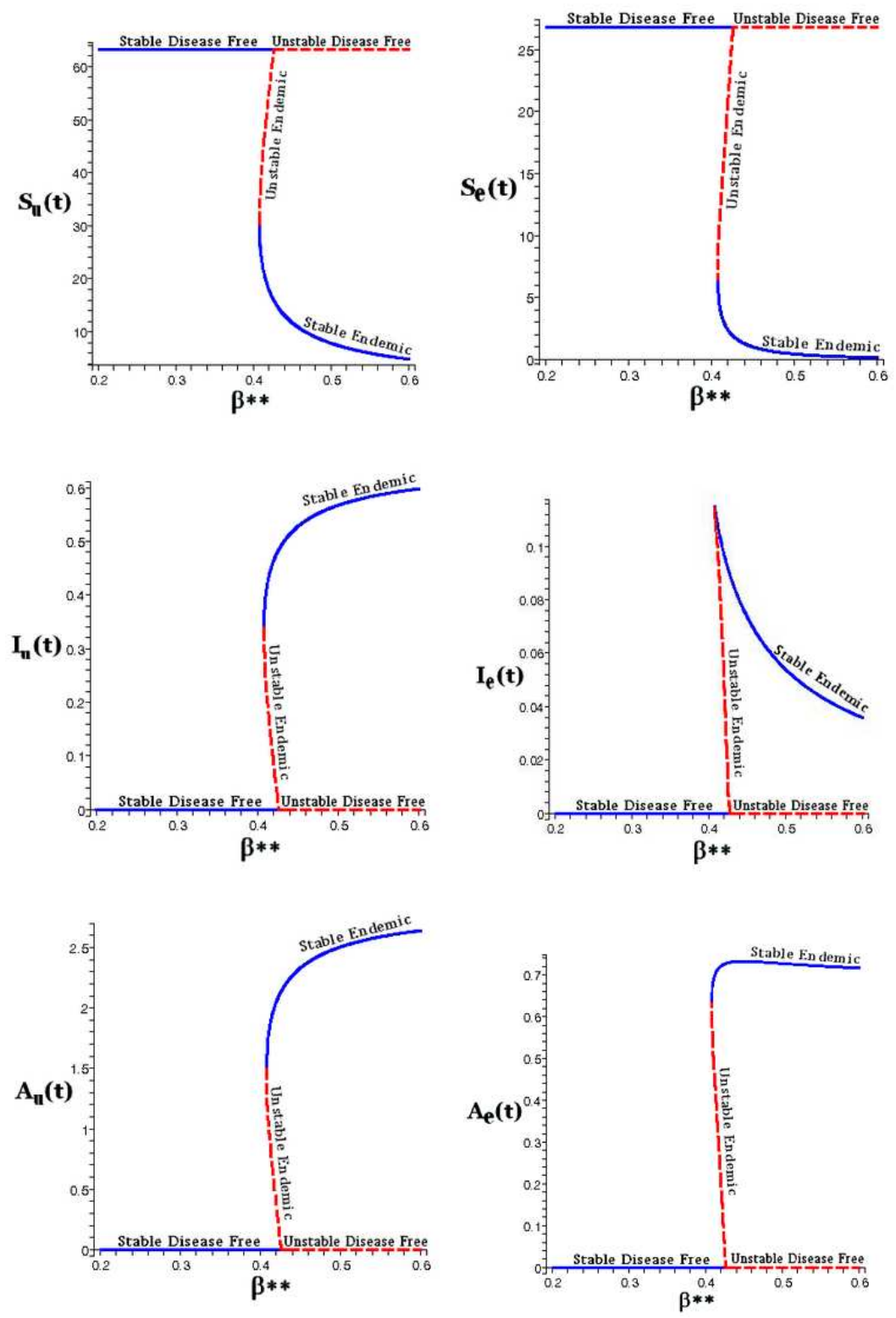

Figure 7: Backward bifurcation diagrams using demographic data from Ethiopia. Parameter values used are as in Table 2 and 3 with $\xi=0.01, p=\psi_{1}=\psi_{2}=0.001$ and $\epsilon=0.4$ (so that, $a=0.02069982715$ and $b=1.930595939$ ). 